\title{
Insulin Promoter Factor-1 Gene Mutation Linked to Early-Onset Type 2 Diabetes Mellitus Directs Expression of a Dominant Negative Isoprotein
}

\author{
Doris A. Stoffers, Violeta Stanojevic, and Joel F. Habener \\ Laboratory of Molecular Endocrinology, Massachusetts General Hospital, Harvard Medical School, and Howard Hughes Medical \\ Institute, Boston, Massachusetts 02114
}

\begin{abstract}
The homeodomain transcription factor insulin promoter factor-1 (IPF-1) is required for development of the pancreas and also mediates glucose-responsive stimulation of insulin gene transcription. Earlier we described a human subject with pancreatic agenesis attributable to homozygosity for a cytosine deletion in codon 63 of the IPF-1 gene (Pro63fsdelC). Pro63fsdelC resulted in the premature truncation of an IPF-1 protein which lacked the homeodomain required for DNA binding and nuclear localization. Subsequently, we linked the heterozygous state of this mutation with type 2 diabetes mellitus in the extended family of the pancreatic agenesis proband. In the course of expressing the mutant IPF-1 protein in eukaryotic cells, we detected a second IPF-1 isoform, recognized by $\mathrm{COOH}$ - but not $\mathrm{NH}_{2}$-terminal-specific antisera. This isoform localizes to the nucleus and retains DNA-binding functions. We provide evidence that internal translation initiating at an out-of-frame AUG accounts for the appearance of this protein. The reading frame crosses over to the wild-type IPF-1 reading frame at the site of the point deletion just carboxy proximal to the transactivation domain. Thus, the single mutated allele results in the translation of two IPF-1 isoproteins, one of which consists of the $\mathrm{NH}_{2}$-terminal transactivation domain and is sequestered in the cytoplasm and the second of which contains the COOH-terminal DNA-binding domain, but lacks the transactivation domain. Further, the $\mathrm{COOH}-$ terminal mutant IPF-1 isoform does not activate transcription and inhibits the transactivation functions of wild-type IPF-1. This circumstance suggests that the mechanism of diabetes in these individuals may be due not only to reduced gene dosage, but also to a dominant negative inhibition of transcription of the insulin gene and other $\beta$ cell-specific genes regulated by the mutant IPF-1. (J. Clin. Invest. 1998. 102: 232-241.) Key words: mutated allele • internal translation • gene transactivation
\end{abstract}

\footnotetext{
Address correspondence to Joel F. Habener, M.D., Laboratory of Molecular Endocrinology, Wellman 320, Massachusetts General Hospital, 50 Blossom St., Boston, MA 02114. Phone: 617-726-5190; FAX: 617-726-6954; E-mail: habenerj@a1.mgh.harvard.edu

Received for publication 12 November 1997 and accepted in revised form 22 April 1998.
}

J. Clin. Invest.

(C) The American Society for Clinical Investigation, Inc. 0021-9738/98/07/0232/10 \$2.00

Volume 102, Number 1, July 1998, 232-241

http://www.jci.org

\section{Introduction}

Early onset type 2 diabetes mellitus (maturity onset diabetes of the young [MODY $]^{1}$ ) is an autosomal dominant disease characterized by the development of non-insulin-dependent diabetes mellitus at an early age (1). To date, four genetic defects that cause MODY monogenic form of type 2 diabetes have been uncovered, three of which correspond to transcription factors expressed in pancreatic $\beta$ cells (hepatic nuclear factor $[\mathrm{HNF}] 1 \alpha$ and $\mathrm{HNF} 4 \alpha$, and insulin promoter factor-1 $[\mathrm{IPF}-1])^{2}(2-4)$. Recently, a mutation in another transcription factor, HNF1 $\beta$, has been associated with MODY (5). The homeodomain transcription factor IPF-1 (also known as IDX-1/ STF-1/PDX-1) is required for development of the pancreas $(6,7)$ and also mediates glucose-responsive stimulation of insulin gene transcription (8-10). IPF-1 is also implicated in the transcriptional regulation of other $\beta$ cell-specific genes, including glucose transporter-2, glucokinase, and islet amyloid polypeptide (11-13). Transcriptional activation of somatostatin by IPF-1 depends on an $\mathrm{NH}_{2}$-terminal transactivation domain (14). The synergistic activation of the insulin gene by the bHLH factor E47 and IPF-1 also depends on the $\mathrm{NH}_{2}$-terminal transactivation domain of IPF-1 (15) and IPF-1 can rescue glucocorticoid-mediated suppression of an insulin transcriptional reporter in HIT cells (16).

In an earlier report, we described a child in whom the pancreas did not develop (pancreatic agenesis) and who was also homozygous for an inactivating cytosine deletion mutation in the protein coding sequence of the IPF-1 gene (Pro63fsdelC) (7). Subsequently, we showed that, in heterozygous carriers of the mutant IPF-1 allele within both branches of the extended family of the proband, the mutation is linked to autosomal dominant early onset type 2 diabetes mellitus (MODY4) (4).

In our earlier report we described the Pro63fsdelC mutation as an inactivating mutation resulting in the premature termination of the translation of IPF-1 (7). This premature termination prevented the translation of the $\mathrm{COOH}$-terminal domain of the protein consisting of the homeodomain that contains the DNA-binding and nuclear translocation functions (7). We reported that a polyclonal antiserum raised against a GST-IDX-1 fusion protein (Hm66) did not detect the prematurely terminated cytoplasmic mutant protein nor any other mutant protein forms on Western blot analysis (7). Subsequent development and characterization of an antiserum specific for the COOH terminus of IPF-1 (see Fig. 1) has revealed that the

1. Abbreviations used in this paper: EMSA, electrophoretic mobility shift assay; HNF, hepatic nuclear factor; IPF-1, insulin promoter factor-1; MODY, maturity onset diabetes of the young; mut, mutant; ORF, open reading frame; wt, wild-type.

2. The officially designated name for the human gene locus for this gene is IPF-1 (Genome Database Nomenclature Committee, University College, London). 
WT

$\alpha \mathbf{N}$

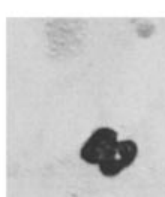

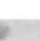$$
\text { T }
$$
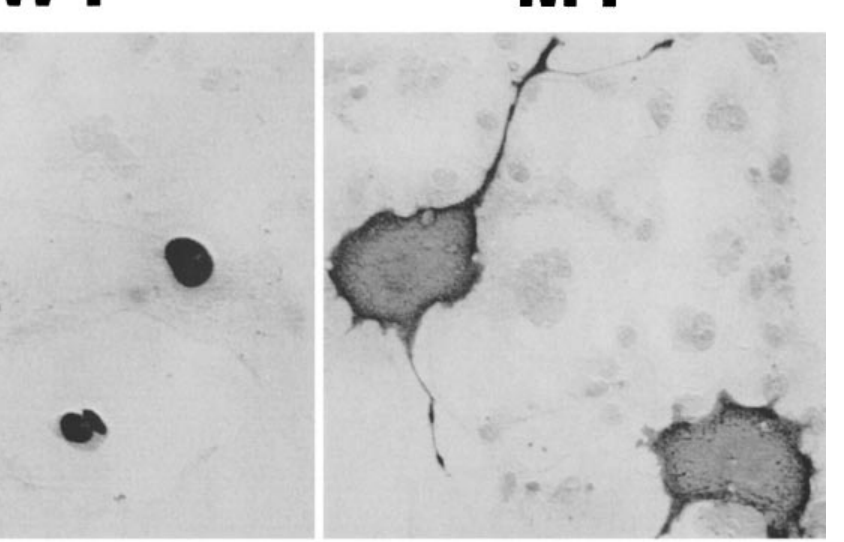

$\alpha \mathrm{C}$
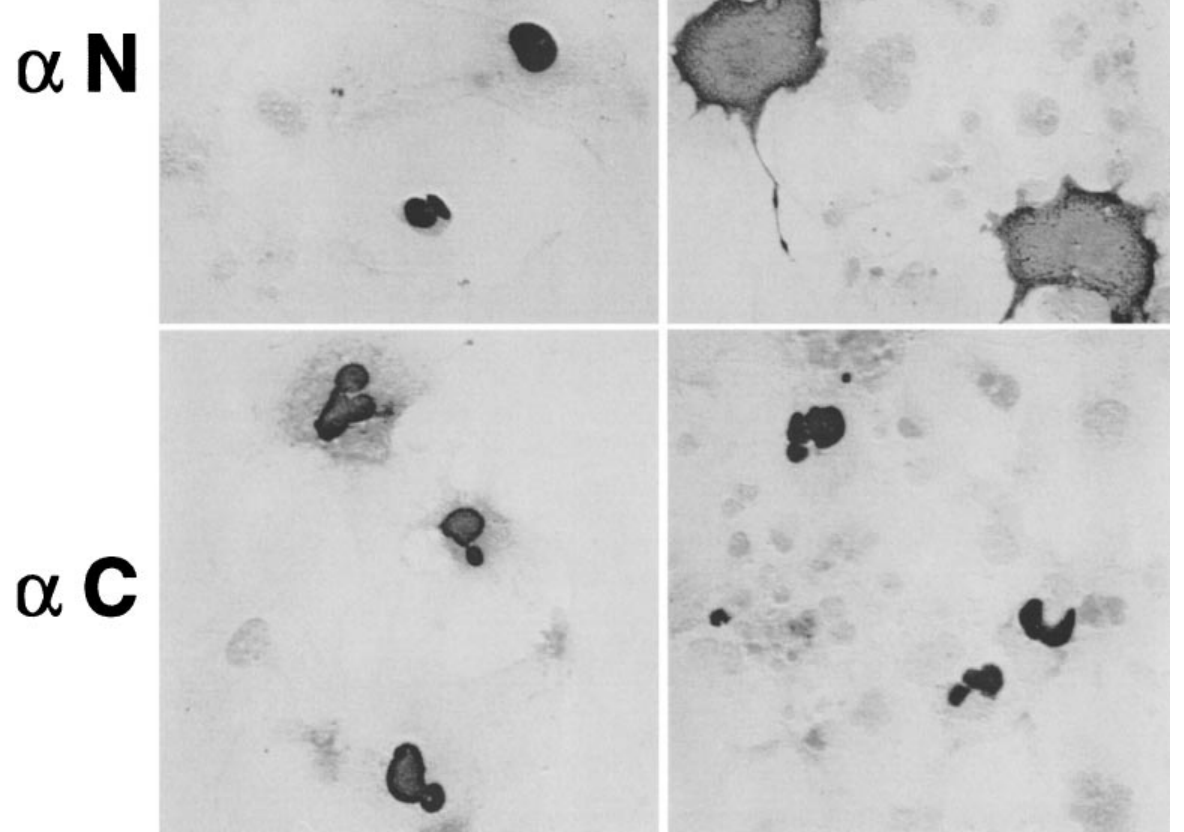

Figure 1. (A) Immunostaining of Cos-1 cells transfected with mut IPF-1 expression plasmid shows nuclear localization of mut IPF-1. h/rwt and h/rmut IPF-1 expression plasmids were transfected into Cos- 1 cells. Fixed cells were stained with $\mathrm{NH}_{2}-(\alpha \mathrm{N})$ and $\mathrm{COOH}$-terminal $(\alpha \mathrm{C})$-specific antisera. $(B)$ EMSAs show complexes between the Far FLAT DNA element of the rat insulin 1 promoter and mut IPF-1 in nuclear extracts of BHK cells transfected with mut IPF-1 expression plasmid. (Left) EMSA and supershift analysis; free probe (lane 1 ), BHK cells transfected with hwt IPF-1 (lanes 2-4), hmut IPF-1 (lanes 5-7), and pcmv5 (empty expression vector; lanes $8-10)$. Antisera were preimmune ( $P I I$; lanes 2,5 , and 8 ), COOH-terminal-specific IPF-1 $(\alpha \mathrm{C}$; lanes 3,6 , and 9 ), or $\mathrm{NH}_{2}$-terminal specific IPF-1 $(\alpha \mathrm{N}$; lanes 4, 7, and 10). Supershifts (SS), wt IPF-1 and mut IPF-1 complexes, and free probe $(F P)$ are indicated by arrows. Nonspecific complexes also present in empty vector transfected BHK cells are denoted by asterisks. (Right) Competition studies; free probe (lane 1), hwt IPF-1 (lanes 2-4), hmut IPF-1 (lanes 5-7), and pcmv5 (empty expression vector; lanes $8-10$ ). The specific competitor was the Far FLAT element (lanes 3,6, and 9). The nonspecific competitor was the somatostatin CRE (lanes 4, 7, and 10). Competitors were included in the binding reaction in $\sim 400$-fold excess. $(C)$

Western blot of nuclear extracts prepared from BHK cells transfected with pcmv5 (empty vector, lanes 1 and 4), hwt IPF-1 (lanes 2 and 5), and hmut IPF-1 (lanes 3 and 6 ) expression plasmid and immunoblotted with COOH- $\left(\alpha \mathrm{C}\right.$; left) and $\mathrm{NH}_{2}$-terminal-specific $(\alpha \mathrm{N} ;$ right) IPF-1 antisera. $40 \mu \mathrm{g}$ of nuclear extract was loaded in each lane.

mutant IPF-1 allele also encodes a protein that translocates to the nucleus and binds to an IPF-1-specific DNA control element in the rat insulin 1 gene promoter. We describe herein the evidence for the existence of such an IPF-1 isoform lacking the transactivation domain and characterize the functional effect of this isoform on two IPF-1 target genes.

\section{Methods}

Vectors. Construction of expression vectors containing wild-type (wt) and mutant (mut) IPF-1 harboring the Pro63fsdelC mutation was described previously (7). These cDNAs are fusions of human exon 1 from a normal individual or an individual harboring the Pro63fsdelC mutation with exon 2 of rat IDX-1. These were cloned into the expression vector pcmv5. Subsequently, we received an intact human IPF-1 cDNA amplified from human 13-wk fetal pancreas as a gift from Henk-Jan Anstoot and Aart Verwest (Sophia Children's Hospital, Rotterdam, The Netherlands); this cDNA was also transferred into the pcmv5 eukaryotic expression vector and the Pro63fsdelC mutation was introduced by site-directed mutagenesis (Quik-Change kit; Stratagene, La Jolla, CA). The names of the plasmids derived from the human/rat parental vector are designated with $\mathrm{h} / \mathrm{r}$ and the fully human IPF- 1 constructs are designated by names beginning with $\mathrm{h}$. The IPF- 1 proteins encoded by $\mathrm{h} / \mathrm{r}$ and $\mathrm{h}$ plasmids behave similarly in electrophoretic mobility shift assays (EMSAs), Western, immunostaining and transactivation assays. A construct lacking the translation initiation site for wt IPF-1 was created through the use of a unique EcoRV site in the 5' pcmv5 polylinker and the unique ScaI site positioned at nucleotide 19 of ORF-1 (see Fig. $2 A$ ) between the wt IPF-1 and the out-of-frame initiator methionines. The EcoRV-ScaI fragment was removed from the h/rmut IPF-1 expression construct and the remaining fragment was religated to create h/rmut IPF-1 $\triangle$ AUG. The reporter constructs were the multimerized somatostatin TAAT1 element reporter vector, SMS(TAAT1) $5^{-}$ 65SMS CAT (17), and the multimerized rat insulin I promoter Far FLAT enhancer (5FF1CAT) (18).

Antisera. The IPF-1 $\mathrm{NH}_{2}$-terminal antiserum $(\alpha \mathrm{N})$ and $\mathrm{COOH}$ terminal $(\alpha \mathrm{C})$ antiserum were raised in rabbits against the first and last 12 amino acids of rat IDX-1, respectively (7). A new antiserum $\left(\alpha \mathrm{N}_{\text {mut }}\right.$; see Fig. 2 B) was generated against the first 12 amino acids of the unique $\mathrm{NH}_{2}$ terminus of the alternatively translated IPF- 1 open reading frame, amino acid sequence RVPARPGAGVQR.

Immunocytochemistry. Transfected cells were trypsinized and seeded into 4-well chamber slides (Nunc Inc., Naperville, IL), allowed to reattach for $24 \mathrm{~h}$, and stained as described (7). Primary antisera were used at 1:750 dilution.

Expression studies. Transfections into Cos-1 cells were performed using DEAE-dextran and a brief dimethylsulfoxide shock (19). BHK cells were transfected with lipofectin (GIBCO BRL, Gaithersburg, MD). RIN5AH cells were transfected by DEAE-dextran in solution suspension (20). NIH 3T3 and HeLa cells were transfected using the calcium phosphate method (5 Prime $\rightarrow 3$ Prime, Inc., Boulder, CO). CAT assays were performed using FAST CAT as substrate, and visualized and quantitated on a Fluorimager (Molecular Dynamics, Sunnyvale, CA). In all transfections, equal amounts of mut and wt IPF-1 expression vectors were used.

Western blot analysis. Western blot analysis of nuclear extracts 
B
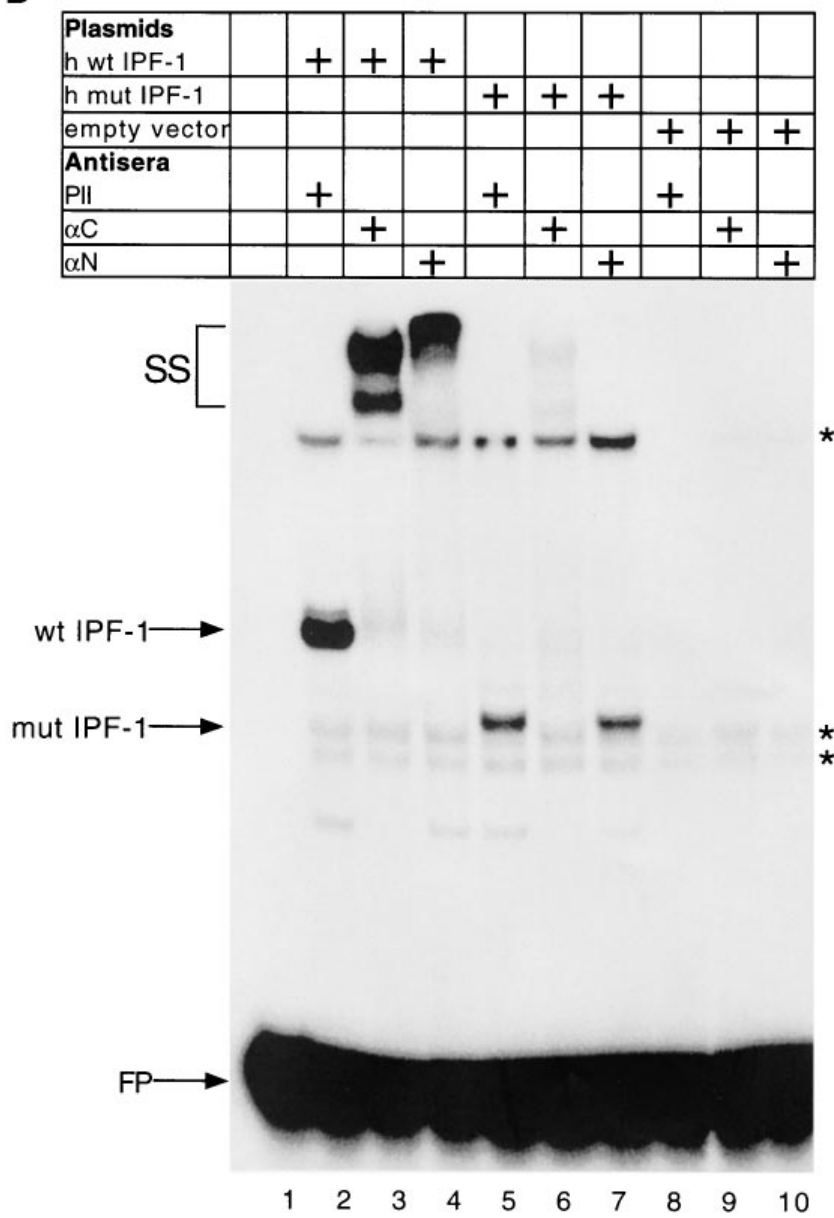

Figure 1 (Continued)

was performed as described (7). The $\mathrm{NH}_{2}$ - and $\mathrm{COOH}$-terminal antisera were used at 1:10,000 dilution, whereas the antiserum to the alternate reading frame $\left(\alpha \mathrm{N}_{\mathrm{mut}}\right)$ was used at 1:500.

Site-directed mutagenesis. Site-directed mutagenesis of the outof-frame AUG beginning at nucleotide 51 of the wt IPF-1 open reading frame (ORF-1, see Fig. $2 A$ ) was performed by substituting A with $\mathrm{G}$ at nucleotide $51 \mathrm{in} \mathrm{h} / \mathrm{rmut}$ IPF-1 using the Quik-Change kit (Stratagene). This mutation converted the methionine codon (AUG) to a valine codon (GUG) in ORF-3 while leaving the amino acid coding sequence of the $\mathrm{NH}_{2}$-terminal truncated translation product (mut IPF-1 N isoform) unchanged [CCA (Pro 17) $\rightarrow$ CCG (Pro 17) in ORF-1].

EMSAs. EMSAs were performed with the Far FLAT enhancer of the rat insulin 1 gene promoter as the ${ }^{32} \mathrm{P}$-labeled oligonucleotide probe (sequence 5'-GATCCTTCATCACGGCATCTGGCCCCTTGTTAATAATCTAATTACCCTAGGTCTA-3') using previously described methods (17). IPF-1 has been shown to be the predominant
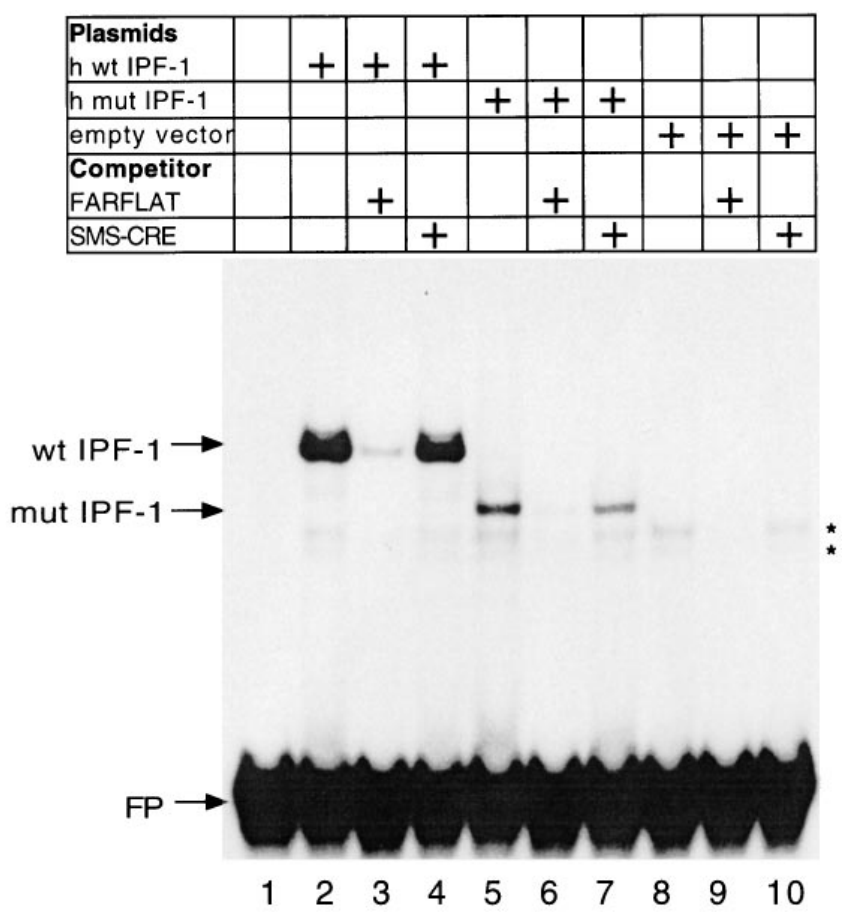

C

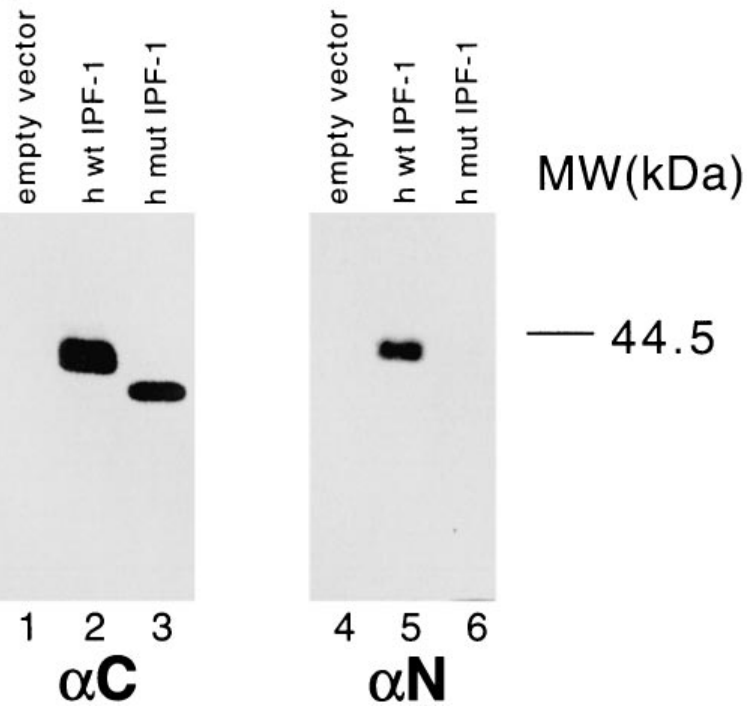

transcription factor binding to this element (15). Nuclear extracts were prepared from BHK cells transfected with either wt or mut IPF-1 expression vectors. Supershifts were performed by adding $1 \mu l$ of polyclonal antiserum for $15 \mathrm{~min}$ before the addition of labeled oligonucleotide. Complexes were resolved on $6 \%$ nondenaturing polyacrylamide gels. In competition studies, the specific competitor was the unlabeled rat insulin 1 promoter Far FLAT element (see above) and the nonspecific competitor was the unlabeled somatostatin CRE element (sequence: 5'-GATCCGGCGCCTCCTTGGCTGACGTCAGAGAGAGAGA-3') (20).

\section{Results}

Detection of a COOH-terminal immunoreactive mut IPF-1 isoform. Development of an antiserum specific for the $\mathrm{COOH}$ terminus of wt IPF-1 revealed that, in addition to the truncated 
A
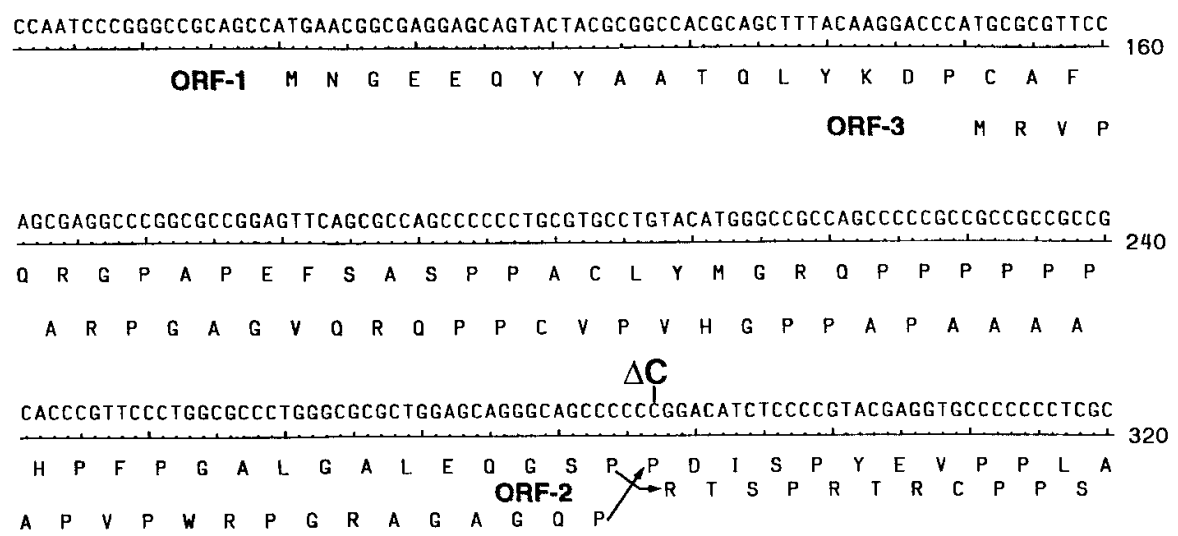

CGACGACCCCGCGgTGGCGCACCTTCACCACCACC TCCCGGCTCAGCTCGCGCTCCCCCACCCGCCCGCCGGGCCCTTCC

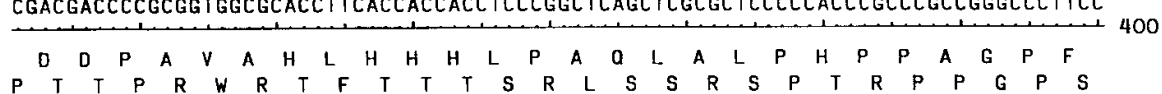
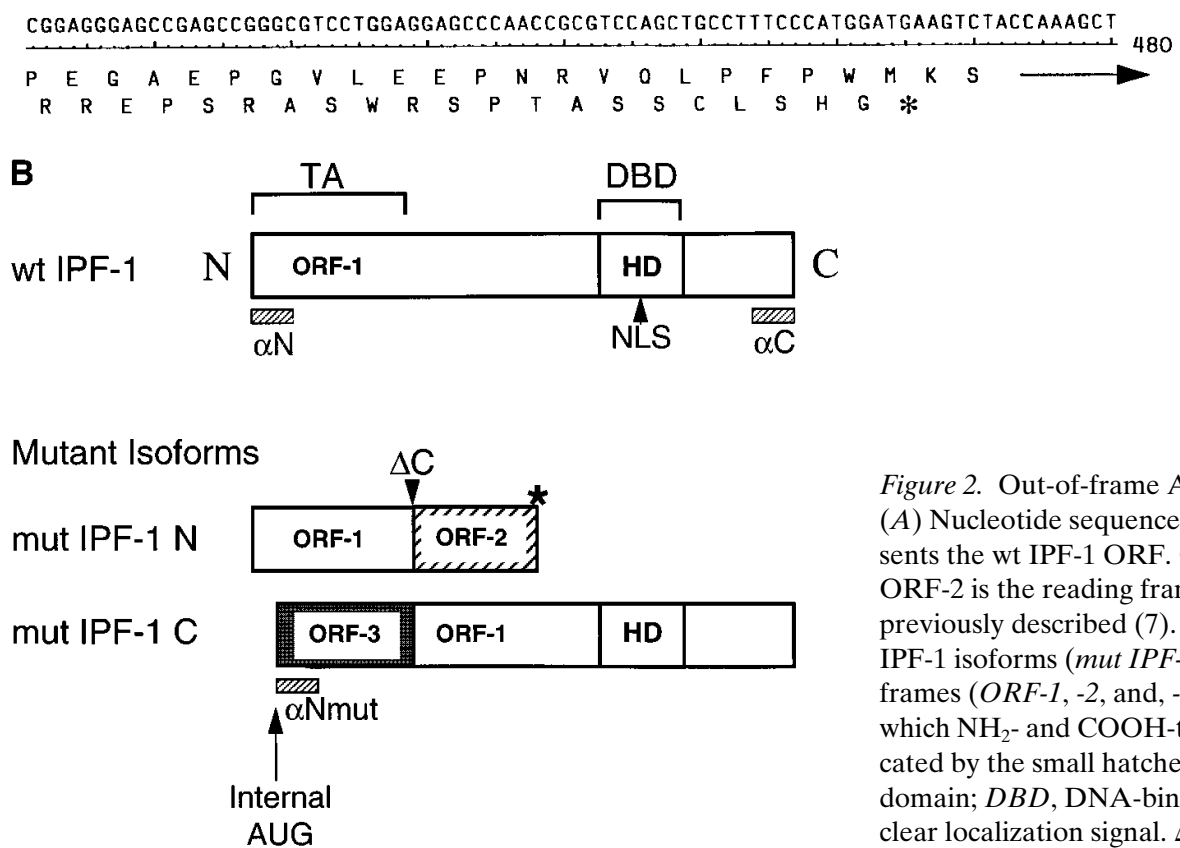

Figure 2. Out-of-frame AUG noted with cross-over of reading frames. $(A)$ Nucleotide sequence of exon 1 with reading frames. ORF-1 represents the wt IPF-1 ORF. ORF-3 initiates at nucleotide 51 of ORF-1. ORF-2 is the reading frame that IPF-1 leads into after Pro63fsdelC as previously described (7). (B) Schematic depiction of proposed mut IPF-1 isoforms (mut IPF-1 N and mut IPF-1 C) and open reading frames $(O R F-1,-2$, and,-3$)$. The positions of the synthetic peptides to which $\mathrm{NH}_{2}$ - and $\mathrm{COOH}$-terminal-specific antisera were raised are indicated by the small hatched bars $(N, C$, and $N m u t)$. TA, Transactivation domain; $D B D$, DNA-binding domain; $H D$, homeodomain; $N L S$, nuclear localization signal. $\Delta \mathrm{C}$ marks the position of Pro63fsdelC.

cytoplasmic $\mathrm{NH}_{2}$-terminal isoform previously described (6), the mut IPF-1 allele also encodes a protein that translocates to the nucleus (Fig. $1 A$ ) and binds to an IPF-1-specific DNA control element in the rat insulin 1 gene promoter (15) (Fig. 1 $B$, left). This $\mathrm{COOH}$-terminal immunoreactive isoform was not detected previously because the Hm66 antiserum does not recognize this isoform of IPF-1 on Western blots. However, the Hm66 antiserum does recognize the $\mathrm{COOH}$-terminal isoform on immunostaining and in supershifts on EMSA (data not shown), suggesting the existence of a specific conformational effect preventing visualization on Western blots.

Our studies used two types of expression vectors for IPF-1, a human exon 1/rat exon 2 fusion IPF-1 cDNA (h/r IPF-1) described previously (7) and an intact human IPF-1 cDNA amplified from human fetal pancreatic cDNA (h IPF-1). The hu-

man and rat homologues of IPF-1 share $100 \%$ amino acid identity in the homeodomain DNA-binding domain and high conservation in the flanking domains. Here and in the previous report (7), we demonstrate that the h/rwt IPF-1 fusion protein retains all properties compared with rat IDX-1 and human IPF-1 in terms of DNA binding, subcellular localization, transactivation, and with regard to the generation of a $\mathrm{COOH}$-terminal immunoreactive IPF-1 isoform in the context of the Pro63fsdelC mutation.

Cross-over of reading frames in the setting of Pro63fsdelC. The unanticipated findings of a $\mathrm{COOH}$-terminal immunoreactive isoform of IPF-1 prompted us to examine more closely the nucleotide sequence of exon 1 of IPF-1 for the possibility of an internal reinitiation of translation of the mutated IPF-1 mRNA triggered by the premature termination of translation. 


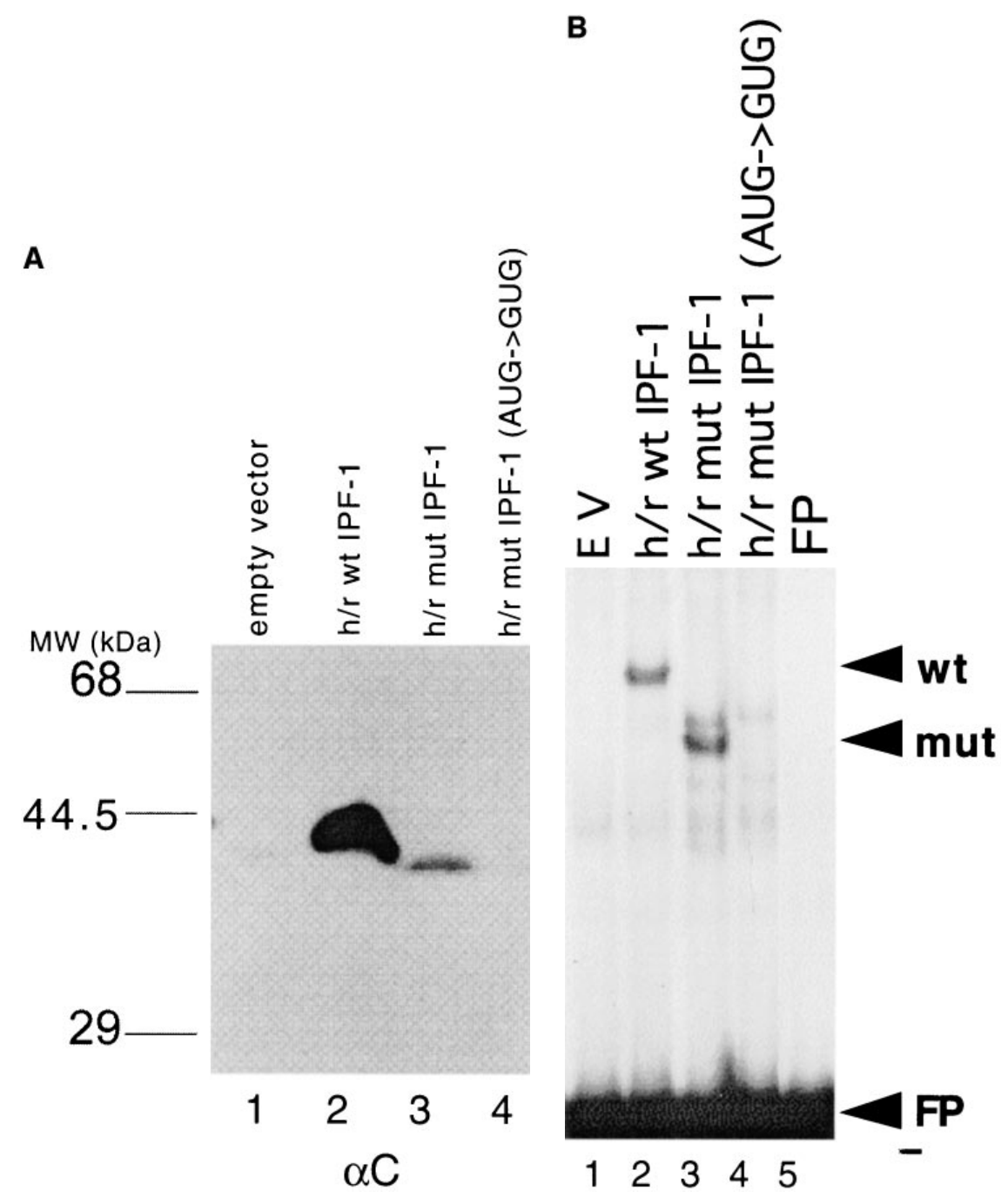

Figure 3. Site-directed mutagenesis of nucleotide 51 to eliminate the alternative start codon. (A) Western blot analysis of whole cell extracts prepared from BHK cells transfected with the indicated expression plasmids, empty vector (lane 1), h/rwt IPF-1 (lane 2), h/rmut IPF-1 (lane 3), and h/rmut IPF-1 (AUG $\rightarrow$ GUG) (lane 4). An equal volume of whole cell lysate was loaded in each lane. The $\mathrm{COOH}$ terminal-specific IPF-1 antiserum was used $(\alpha C)$. (B) EMSA analysis of nuclear extracts prepared from BHK cells transfected as in $A$ and incubated with the rat insulin Far FLAT element as the labeled oligonucleotide probe.
Upon examination of the nucleotide sequence of the IPF-1 mRNA we found an AUG codon in a reasonable context for translational initiation at nucleotide 51 of the wt IPF-1 open reading frame (21) (ORF-1, Fig. $2 A$ ). Although translation is out of the reading frame for wt IPF-1, the mutation Pro63fsdelC in the mut IPF-1 allele shifts and reestablishes the correct reading frame for the protein (Fig. 2, $A$ and $B$ ). The consequence of this translational frame-shift is the synthesis of an alternative isoform of IPF-1 (mut IPF-1 C) that lacks the $\mathrm{NH}_{2}$-terminal domain critical for the transactivation of gene transcription $(14,15)$ but retains the DNA-binding and nuclear translocation signals (schematically depicted in Fig. 2 B). Consistent with this prediction, the mut IPF-1 DNA complex detected in EMSA assays is shifted with the COOH-terminalspecific IPF-1 antiserum but not the $\mathrm{NH}_{2}$-terminal-specific antiserum (Fig. $1 \mathrm{~B}$ ). Similarly, on Western immunoblots, this IPF-1 isoform is not detected by the $\mathrm{NH}_{2}$-terminal-specific IPF-1 antiserum (Fig. $1 C$ ).

Effect of site-directed mutagenesis of the out-of-frame AUG at nucleotide 51 of ORF-1. To test the hypothesis that translational initiation from the internal AUG accounts for mut IPF-1 C observed on Western blot, EMSA, and immunofluorescence, we mutated this AUG to GUG by site-directed mu- tagenesis to encode a valine (ORF-3, Fig. $2 A)$, without altering the amino acid sequence of mut IPF-1 N (ORF-1, Fig. $2 A$ ) (see Methods). Expression of the AUG to GUG mutated IPF-1 resulted in a loss of the expression of the $42-\mathrm{kD} \mathrm{COOH}$-terminal immunoreactive protein in transfected BHK cells (Fig. $3 A$, lane 4) indicating that reinitiation of translation of the mut IPF-1 mRNA occurs at this AUG codon. Furthermore, the specific complex observed in EMSA experiments is also eliminated through the mutagenesis of the AUG beginning at nucleotide 51 (Fig. 3 B, lane 4).

Immunological verification of the $\mathrm{NH}_{2}$-terminal reading frame of the Pro63fsdelC COOH-terminal isoform. To verify the reading frame of the $\mathrm{NH}_{2}$ terminus of mut IPF-1 C, we generated an antiserum specific for the $\mathrm{NH}_{2}$ terminus of ORF-3 ( $\alpha$ Nmut) (Fig. $2 B$ ). This antiserum specifically recognized mut IPF-1 C (Fig. $4 A$, lanes 6 and 9 ) but not wt IPF-1 (lanes 5 and 8 ). Furthermore, the complex between mut IPF-1 C and Far FLAT DNA is specifically supershifted by $\alpha$ mut (Fig. $4 B$, lane 5), whereas the wt IPF-1 complex is unaffected (lane 3). To determine whether this alternate reading frame is used under normal in vivo conditions, we used the $\alpha$ Nmut antiserum on a Western blot of whole cell extracts from human islets, HeLa, and Capan-1 cells. No immunoreactive proteins at or 
A

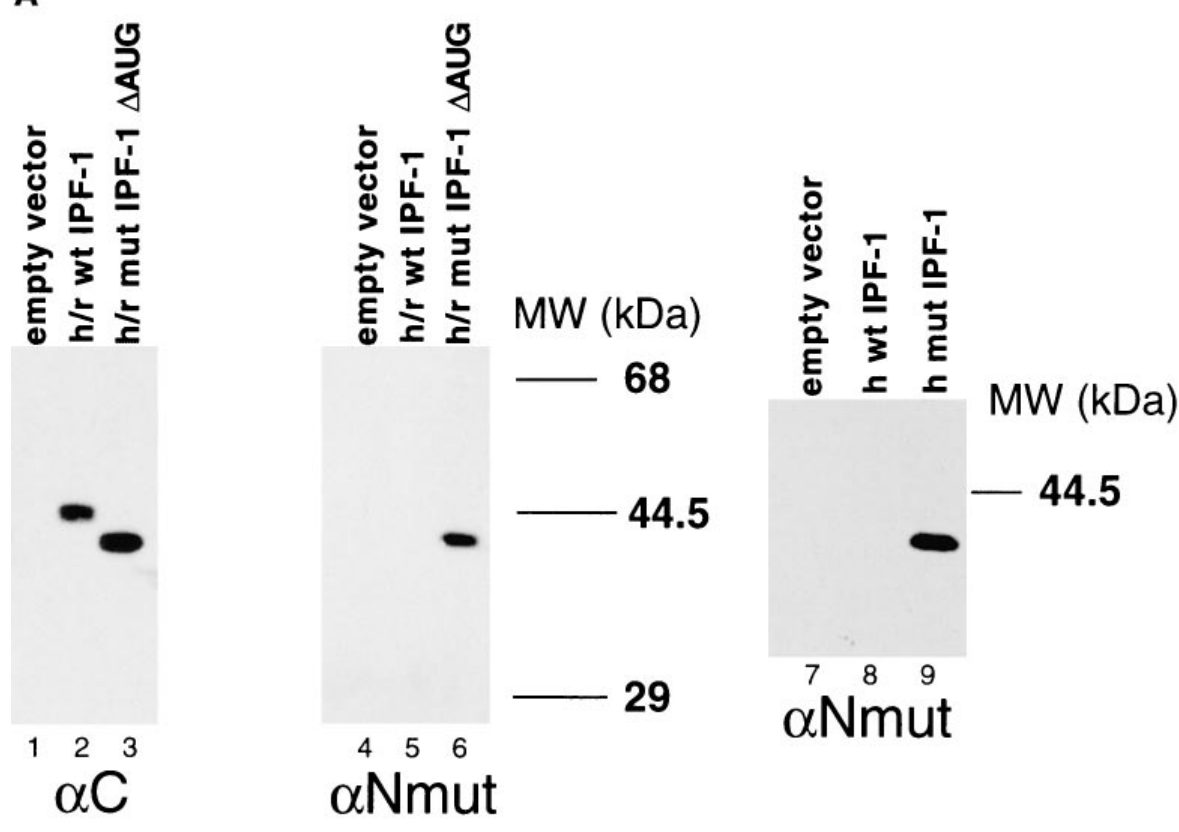

Figure 4. Verification of the $\mathrm{NH}_{2}$-terminal reading frame of the $\mathrm{COOH}$-terminal immunoreactive mut IPF-1 isoform. $(A)$ Western blot analysis of nuclear extracts prepared from BHK cells transfected with empty vector (lanes 1,4 , and 7), h/rwt IPF-1 (lanes 2 and 5), h/rmut IPF-1 $\Delta$ AUG (lanes 3 and 6), hwt IPF-1 (lane 8), and hmut IPF-1 (lane 9) and visualized with $\alpha \mathrm{C}$ (left) and $\alpha \mathrm{N}_{\mathrm{mut}}$ antisera (middle and right). An equal volume of whole cell lysate was loaded in each lane of the left and middle panels (lanes 1-6). $40 \mu \mathrm{g}$ of nuclear extract was loaded in the right panel (lanes 7-9).

(B) EMSA of nuclear extract of BHK cells transfected with empty vector (lanes 6 and 7), hwt IPF-1 (lanes 2 and 3), and hmut IPF-1 (lanes 4 and 5). All lanes contained $1 \mu \mathrm{l}$ of either preimmune antiserum (lanes 2, 4, and 6) or $\alpha \mathrm{N}_{\mathrm{mut}}$ antiserum (lanes 3, 5, and 7). $F P$, Free probe. $(C)$ The start AUG codon at nucleotide 51 is used by an insulinoma cell line. Western immunoblot of RIN 5AH cells transfected with empty vector (lane 1), h/rwt IPF-1 (lane 2), and

h/rmut IPF-1 $\triangle$ AUG (lane 3) visualized with IPF-1 COOH-terminal-specific antiserum $\left(\alpha \mathrm{C}\right.$; left) and the alternate IPF-1 $\mathrm{NH}_{2}$-terminal-specific antiserum $(\alpha \mathrm{Nmut}$; right). $10 \mu \mathrm{g}$ of nuclear extract was loaded in each lane.

around the predicted molecular mass of ORF-3 $(21.3 \mathrm{kD})$ were observed (data not shown).

Translation initiation site selection in a $\beta$ cell insulinoma cell line. To obtain higher levels of expression and to verify that the AUG at nucleotide 51 is suitable for translation initiation, we created a construct harboring Pro63fsdelC but lacking the translation initiation codon for wt IPF-1 (h/rmut IPF-1 $\Delta$ AUG). This resulted in translation of a protein of the same molecular weight and immunoreactivity as mut IPF-1 C but at levels comparable to the expression levels of wt IPF-1 (Fig. 4 $A$, lane 3 ). This construct was transfected into the RIN5AH insulinoma $\beta$ cell tumor cell line. Endogenous IPF-1 could be detected (Fig. $4 C$, all lanes). Overexpression of IPF-1 was evident when the wt construct was transfected (Fig. $4 C$, lane 2). Similarly, mut IPF-1 C was detected when the h/rmut IPF-1 $\triangle$ AUG expression plasmid was introduced (lane 3), indicating that the AUG at nucleotide 51 of ORF- 1 is an efficient translation initiator in a $\beta$ cell line. Mut IPF-1 C in RIN5AH cells was also specifically recognized by $\alpha$ Nmut (Fig. $4 C$, lane 6 ).

Function of the alternate IPF-1 isoform. The lack of a transactivation domain raised the likely possibility that the internally translated $\mathrm{COOH}$-terminal immunoreactive IPF-1 isoform would be incapable of activating transcription from IPF-1 target genes. In accordance with this possibility, the mutant isoform expressed from $\mathrm{h} / \mathrm{rmut} \mathrm{IPF}-1$ is unable to transactivate from the somatostatin TAAT1 element, an established binding site and target gene for IPF-1 $(17,22)$. IPF-1 activates the SMS(TAAT1) $)_{5}$-65SMSCAT reporter construct $\sim$ 14-fold compared with 2-fold for mut IPF-1 (Fig. $5 A$ ). mut IPF-1 does not significantly activate the SMS(TAAT1) $)_{5}$-65SMSCAT reporter in the $\beta$ cell line, RIN5AH, even when expressed at high levels from the $\mathrm{h} / \mathrm{rmutIPF}-1 \Delta \mathrm{AUG}$ expression plasmid (Fig. 5 B). Further, mut IPF-1C does not synergize with E47 to activate the multimerized Far FLAT enhancer of the rat insu- lin 1 gene (5FF1CAT) reporter in HeLa cells (Fig. 5C). When increasing amounts of mut IPF-1 are cotransfected with wt IPF-1 in this synergy assay, a progressive inhibition of synergy is observed (Fig. 5 C). Expression of both normal and mut IPF-1 was confirmed by Western blot analysis of the nuclear pellet of transfected cell lysates (data not shown). Thus, the mut IPF- 1 isoform is not only unable to activate transcription, but it exerts a dominant negative effect on the activation of target gene transcription by wt IPF-1.

\section{Discussion}

Here, we demonstrate that a heterozygous mutation in IPF-1 associated with early onset type 2 diabetes mellitus results in the translation of two IPF-1 isoforms from the mutant allele. One isoform consists of the $\mathrm{NH}_{2}$-terminal transactivation domain and is transcriptionally inactive and the second isoform contains the $\mathrm{COOH}$-terminal DNA-binding domain, but lacks the transactivation domain. These isoforms arise in the context of a cytosine deletion in the open reading frame encoded by exon 1. The $\mathrm{COOH}$-terminal isoform results from "backscanning" reinitiation of translation as a result of premature termination of translation of ORF-1. Occurrences of the reinitiation of translation of mRNAs in which the initial translation is prematurely terminated have been reported previously. It is conjectured that the termination-induced release of ribosomes initiated by $5^{\prime}$ upstream AUG start codons relieves ribosomal elongation occlusion and allows for the reinitiation of translation at cryptic internal AUG codons in the mRNA $(23,24)$. Although translational backscanning in eukaryotic mRNAs has been reported (25-27), our findings may be the first example of this mechanism contributing to a disease phenotype.

The existence of an IPF-1 isoform that contains both nuclear localization and DNA binding but no transactivation do- 


\begin{tabular}{|l|l|l|l|l|l|l|l|}
\hline $\begin{array}{l}\text { Plasmids } \\
\text { h wt IPF-1 }\end{array}$ & & + & + & & & & \\
\hline h mut IPF-1 & & & & + & + & & \\
\hline empty vector & & & & & & + & + \\
\hline $\begin{array}{l}\text { Antisera } \\
\text { PII }\end{array}$ & & + & & + & & + & \\
\hline$\alpha$ Nmut & & & + & & + & & + \\
\hline
\end{tabular}

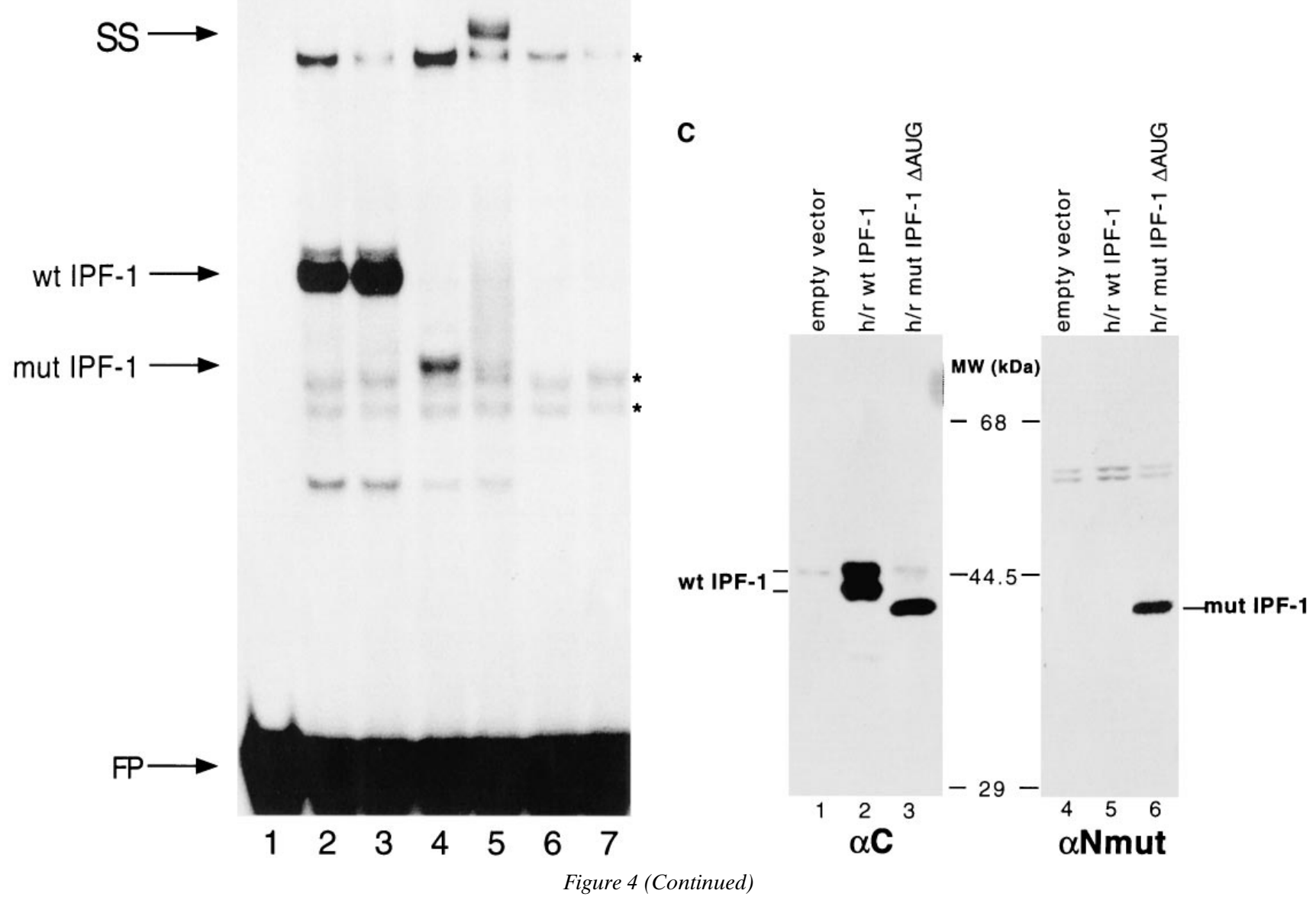

main is relevant to the pathogenetic mechanism of the diabetes associated with this mutation. Initially we considered that a mechanism of decreased gene dosage due to inactivation of one of the two IPF-1 alleles could account for the diabetic phenotype. However, the expression of the $\mathrm{COOH}$-terminal isoform suggests that the mut IPF-1 allele may also serve as a dominant negative inhibitor of the function of the unmutated, wt IPF-1 allele. Several examples of dominant negative isoforms of transcription factors, which lack transactivation domains due to alternative exon splicing of RNA, alternative usage of internal translation codons of mRNA, and genetic mutations have been reported $(23,28,29)$. We show that the mutant COOH-terminal isoform of IPF-1 does not activate the transcription of two different transcriptional reporters that are driven by IPF-1-regulated binding sites. Furthermore, the mut IPF-1 competitively inhibits the ability of wt IPF-1 in synergism with E47 to activate the rat insulin I Far FLAT enhancer. When equal amounts of expression vectors are cotransfected, mut IPF-1 completely abrogates the synergy of wt IPF-1 with
E47 in the activation of the rat insulin I reporter. These findings indicate that even a fraction of mut IPF-1 expression relative to wt IPF-1 in vivo may have a significant inhibitory effect on transcription of IPF-1 target genes.

Additional supportive evidence for our conjecture that the internally translated IPF-1 isoform resulting from the cytosine deletion mutation is unable to transactivate IPF-1 target genes comes from the phenotype of the index subject who is homozygous for this mutation. The subject has pancreatic agenesis. If the mut IPF-1 isoform were to have transactivational activities, one might not expect that the expression of the isoform would result in such a severe phenotype of pancreatic agenesis.

Although the family with the Pro63fsdelC mutation satisfies criteria for MODY, the age of onset of disease was somewhat later than the age of onset for MODY1,2, and 3, suggesting that the IPF-1 deficiency phenotype may represent an intermediate between classic MODY and the more common late onset type 2 diabetes mellitus. The regulation of the rela- 
A
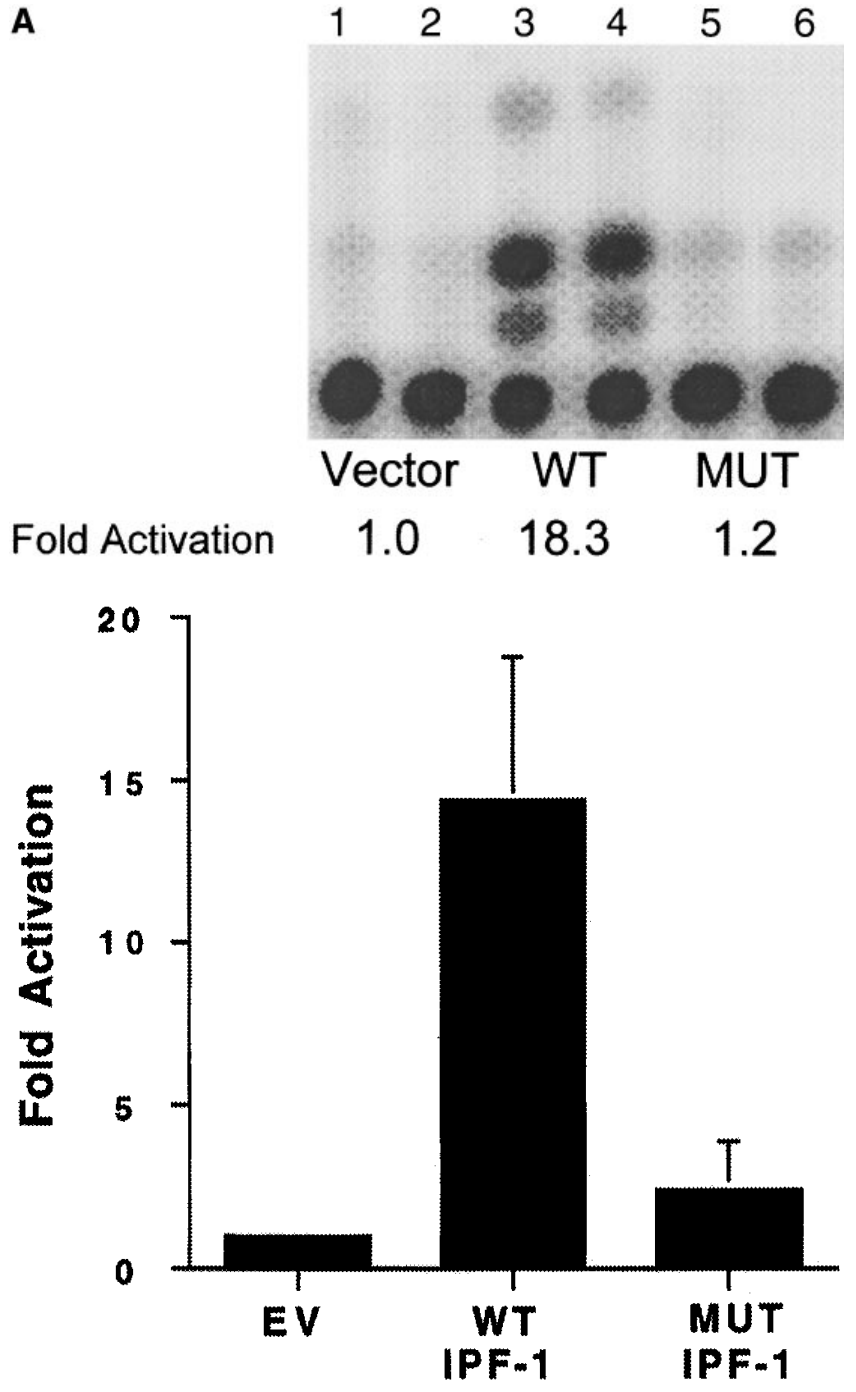

Expression Plasmid

C

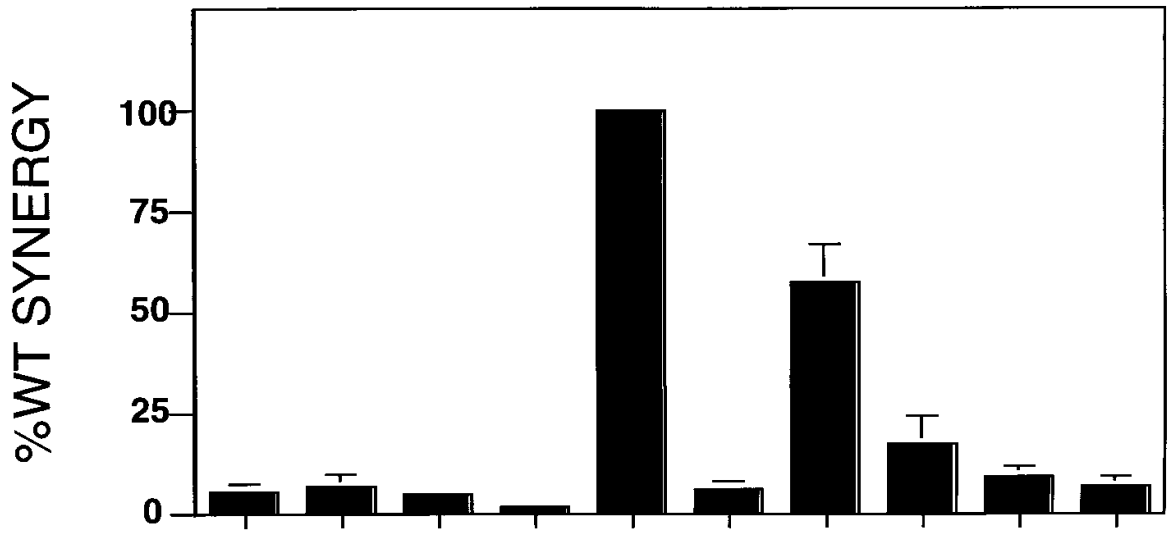

B

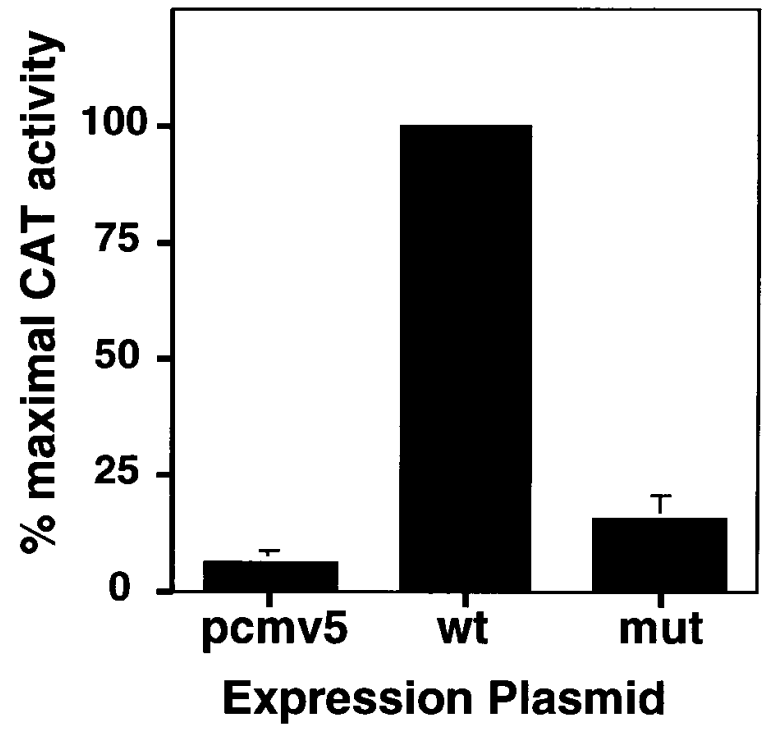

Figure 5. Lack of transactivation and dominant negative effect of mut IPF-1 C. (A) Lack of transactivation function of the mutant allele on a somatostatin promoter reporter construct. (Top) A representative CAT assay in which NIH 3 T3 cells were cotransfected with the somatostatin reporter (SMS TAAT1) $)_{5}$-65SMSCAT and empty vector (lanes 1 and 2), h/rwt IPF-1 (lanes 3 and 4), or h/rmut IPF-1 (lanes 5 and 6) expression plasmid. (Bottom) Mean fold activation over empty vector for three experiments each performed in duplicate or triplicate. Error bars represent SEM $(n=3)$. (B) Lack of transactivation by the expression of mut IPF-1 C (h/rmut IPF-1 $\triangle \mathrm{AUG}$ ) on (SMS TAAT1) $)_{5}$-65SMSCAT reporter in insulin-producing RIN5AH cells. Error bars reflect SEM $(n=5)$. $(C)$ Lack of synergy of h/rmut IPF-1 $\Delta$ AUG with E47 on 5FF1CAT in HeLa cells and dominant negative action on the synergy of h/rwt IPF-1 with E47. Results are expressed as percent maximal synergy (which is that of $\mathrm{h} / \mathrm{rwt}$ IPF-1 with E47). Error bars represent SEM $(n=3)$. The amounts of expression plasmid $(\mu \mathrm{g})$ used in each transfection are indicated. 
tive amounts of the production of IPF-1 from the normal allele and the dominant negative IPF-1 isoform from the mutant allele is unknown; perhaps age will influence the accumulation of the mut IPF-1 isoform relative to the normal IPF-1 gene product. In support of this concept, the deterioration of $\beta$ cell function with age in Wistar rats has been associated with decreased expression of the insulin and GLUT2 genes $(30,31)$, both of which are regulated by IPF-1 $(11,15)$. The levels of IPF-1 as a function of age have not yet been determined; however, it may be relevant that IPF-1 promoter activity appears to diminish with age in transgenic mice bearing the transcriptional reporter lacZ under the control of the IPF-1 promoter (32).

What remains in question, and yet to be determined in the future, are the mechanisms by which the mut IPF-1 leads to the development of diabetes in heterozygous individuals who carry the mutant allele. Clearly, carriers of the inactivating mutation are haploinsufficient for IPF-1, notwithstanding the expression of any dominant negative IPF-1 isoform derived from the expression of the mutant allele. Our transfection expression studies indicate that the mut IPF-1 is expressed at lower levels (10-30\%) compared with the expression of wt IPF-1. It is not unexpected that mut IPF-1 would be relatively unstable when expressed in $\beta$ cells, or other cells, because mutated proteins are often degraded at a faster rate compared with the wild-type, unmutated, protein. However, even a low level of expression of the dominant negative IPF-1 would predictably lower the gene dosage of IPF-1, based on our experimental findings of dominant negative inhibition in transfection expression studies.

It seems reasonable to speculate that over $20-30 \mathrm{yr}$ of a reduced gene dosage effect of IPF-1, the $\beta$ cells may fail to function properly, resulting in relative insulin insufficiency and the development of type 2 diabetes. The failure of $\beta$ cell functions could result from one or more mechanisms, such as failure to adequately stimulate the expression of the insulin, glucokinase, glucose transporter, or other $\beta$ cell specific genes, or even failure to activate genes responsible for the neogenesis of $\beta$ cells required to maintain $\beta$ cell mass in the face of programmed loss of $\beta$ cells by apoptosis (33). Questions remain as to the identities of the relevant target genes in $\beta$ cells regulated by IPF-1.

It is worth noting that four of the five genes responsible for the monogenic forms of MODY and early onset type 2 diabetes encode transcription factors. Further, additional transcription factors important in the development of the endocrine pancreas have been identified by gene knockouts in mice (for review see reference 34 ). One might speculate that mutations in one or more of these genes may also be responsible for the development of monogenic type 2 diabetes in other families.

\section{Acknowledgments}

We are grateful to H. Anstoot and A. Verwest for the human IPF-1 cDNA, T. Kieffer for help with generation of antisera, R. Stein for the E47 expression construct and responsive HeLa cells, and L. Moss for the 5FF1CAT reporter construct. We thank Townley Budde for help in the preparation of this manuscript.

D.A. Stoffers was supported by a Mentored Clinical Scientist Development Award. J.F. Habener is an investigator of the Howard Hughes Medical Institute. These studies were supported in part by U.S. Public Health Service grant DK-30457.

\section{References}

1. Tattersall, R.B., and S.S. Fajans. 1975. A difference between inheritance of classical juvenile-onset and maturity-onset type diabetes of young people. Diabetes. 24:44-53.

2. Yamagata, K., N. Oda, P.J. Kalsaki, S. Menzel, H. Furuta, M. Vaxillaire, L. Southam, R.D. Cox, G.M. Lathrop, V.V. Boriraj, et al. 1996. Mutations in the hepatocyte nuclear factor- $1 \alpha$ gene in maturity-onset diabetes of the young (MODY3). Nature. 384:455-458.

3. Yamagata, K., H. Furuta, N. Oda, P.J. Kaisaki, S. Menzel, N.J. Cox, S.S Fajans, S. Signorini, M. Stoffel, and G.I. Bell. 1996. Mutations in the hepatocyte nuclear factor- $4 \alpha$ gene in maturity-onset diabetes of the young (MODY1). Nature. 384:458-460.

4. Stoffers, D.A., J. Ferrer, W.L. Clarke, and J.F. Habener. 1997. Earlyonset type-II diabetes mellitus (MODY4) linked to IPF-1. Nat. Genet. 17:138139.

5. Horikawa, Y., N. Iwasaki, M. Hara, H. Furuta, Y. Hinokio, B. Cockburn, T. Lindner, K. Yamagata, M. Ogata, O. Tomonaga, et al. 1997. Mutation in hepatocyte nuclear factor-1 $\beta$ gene (TCF2) associated with MODY. Nat. Genet. 17:384-385.

6. Jonsson, J., L. Carlsson, T. Edlund, and H. Edlund. 1994. Insulin-promoter-factor 1 is required for pancreas development in mice. Nature. 371:606609

7. Stoffers, D.A., N.T. Zinkin, V. Stanojevic, W.L. Clarke, and J.F. Habener. 1997. Pancreatic agenesis attributable to a single nucleotide deletion in the human IPF1 coding region. Nat. Genet. 15:106-110.

8. Melloul, D., Y. Ben-Neriah, and E. Cerasi. 1993. Glucose modulates the binding of an islet-specific factor to a conserved sequence within the rat I and the human insulin promoters. Proc. Natl. Acad. Sci. USA. 90:3865-3869.

9. MacFarlane, W., M.L. Read, M. Gilligan, I. Bujalska, and K. Docherty. 1994. Glucose modulates the binding activity of the $\beta$ cell transcription factor IUF-1 in a phosphorylation-dependent manner. Biochem. J. 303:625-631.

10. Marshak, S., H. Totary, E. Cerasi, and D. Melloul. 1996. Purification of the $\beta$-cell glucose-sensitive factor that transactivates the insulin gene differentially in normal and transformed islet cells. Proc. Natl. Acad. Sci. USA. 93: 15057-15062.

11. Waeber, G., N. Thompson, P. Nicod, and C. Bonny. 1996. Transcriptional activation of the GLUT2 gene by the IPF-1/STF-1/IDX-1 homeobox factor. Mol. Endocrinol. 10:1327-1334.

12. Watada, H., Y. Kajimoto, Y. Umayahara, T. Matsuoka, H. Kaneto, Y. Fujitani, T. Kamada, R. Kawamori, and Y. Yamasaki. 1996. The human glucokinase gene $\beta$-cell-type promoter: an essential role of insulin promoter factor 1/PDX-1 in its activation in HIT-T15 cells. Diabetes. 45:1478-1488.

13. Carty, M.D., J.S. Lillquist, M. Peshavaria, R. Stein, and W.C. Soeller. 1997. Identification of cis- and trans-active factors regulating human islet amyloid polypeptide gene expression in pancreatic $\beta$-cells. J. Biol. Chem. 272: 11986-11993.

14. Lu, M., C.P. Miller, and J.F. Habener. 1996. Functional regions of the homeodomain protein IDX-1 required for transactivation of the rat somatostatin gene. Endocrinology. 137:2959-2967.

15. Peers, B., J. Leonard, S. Sharma, G. Teitelman, and M.R. Montminy. 1994. Insulin expression in pancreatic islet cells relies on cooperative interactions between the helix loop helix factor E47 and the homeobox factor STF-1. Mol. Endocrinol. 8:1798-1806.

16. Sharma, S., U.S. Jhala, T. Johnson, K. Ferreri, J. Leonard, and M. Montminy. 1997. Hormonal regulation of an islet-specific enhancer in the pancreatic homeobox gene STF-1. Mol. Cell Biol. 17:2598-2604.

17. Miller, C.P., R. McGehee, and J.F. Habener. 1994. IDX-1: a new homeodomain transcription factor expressed in rat pancreatic islets and duodenum that transactivates the somatostatin gene. EMBO (Eur. Mol. Biol. Organ.) J. 13:1145-1156.

18. German, M.S., L.G. Moss, J. Wang, and W.J. Rutter. 1992. The insulin and islet amyloid polypeptide genes contain similar cell-specific promoter elements that bind identical beta-cell nuclear complexes. Mol. Cell Biol. 12:17771788.

19. Ausubel, F.M., R. Brent, R.E. Kingston, D.D. Moore, J.G. Seidman, J.A. Smith, and K. Struhl. 1995. Current Protocols in Molecular Biology. John Wiley \& Sons, New York.

20. Vallejo, M., L. Penchuk, and J.F. Habener. 1992. Somatostatin gene upstream enhancer element activated by a protein complex consisting of CREB, Isl-1-like, and alpha-CBF-like transcription factors. J. Biol. Chem. 267:1287612884.

21. Kozak, M. 1989. The scanning model for translation: an update. J. Cell Biol. 108:229-241.

22. Leonard, J., B. Peers, T. Johnson, K. Ferreri, S. Lee, and M.R. Montminy. 1993. Characterization of somatostatin transactivating factor-1, a novel homeobox factor that stimulates somatostatin expression in pancreatic islet cells. Mol. Endocrinol. 7:1275-1283.

23. Walker, W.H., C. Girardet, and J.F. Habener. 1996. Alternative exon splicing controls a translational switch from activator to repressor isoforms of transcription factor CREB during spermatogenesis. J. Biol. Chem. 271:2014520150. 
24. Kozak, M. 1995. Adherence to the first-AUG rule when a second AUG codon follows closely upon the first. Proc. Natl. Acad. Sci. USA. 92:2662-2666.

25. Gunnery, S., U. Maivali, and M.B. Mathews. 1997. Translation of an uncapped mRNA involves scanning. J. Biol. Chem. 272:21642-21646.

26. Jackson, R.J. 1996. A comparative view of initiation site selection mechanisms. In Translational Control. J.W.B. Hershey, M.B. Mathews, and N. Sonenberg, editors. Cold Spring Harbor Laboratory Press, Cold Spring Harbor, NY. 71-112.

27. Geballe, A.P. 1996. Translational control mediated by upstream AUG codons. In Translational Control. J.W.B. Hershey, M.B. Mathews, and N. Sonenberg, editors. Cold Spring Harbor Laboratory Press, Cold Spring Harbor, NY. 173-198.

28. Baniahmad, A., S.Y. Tsai, B.W. O’Malley, and M.-J. Tsai. 1992. Kindred $\mathrm{S}$ thyroid hormone receptor is an active and constitutive silencer and a repressor for thyroid hormone and retinoic acid responses. Proc. Natl. Acad. Sci. USA. 89:10633-10637.

29. Radovick, S., M. Nations, Y. Du, L.A. Berg, B.D. Weintraub, and F.E. Wondisford. 1992. A mutation in the POU-homeodomain of Pit-1 responsible for combined pituitary hormone deficiency. Science. 257:1115-1117.

30. Wang, Y., R. Perfetti, N.H. Greig, H.W. Holloway, K.A. DeOre, C. Montrose-Rafizadeh, D. Elahi, and J.M. Egan. 1997. Glucagon-like peptide-1 can reverse the age-related decline in glucose tolerance in rats. J. Clin. Invest. 99:2883-2889.

31. Perfetti, R., C. Montrose-Rafizadeh, A.S. Liotta, and J.M. Egan. 1995 Age-dependent reduction in insulin secretion and insulin mRNA in isolated islets from rats. Am. J. Physiol. 269:E983-E990.

32. Stoffers, D.A., R.S. Heller, C.P. Miller, and J.F. Habener. 1996. Expression of homeodomain factor IDX-1 in pancreas and duodenum: discordance among expression of an IDX-1 $\beta$-galactosidase transcriptional reporter, IDX-1 mRNA and protein. 10th Int. Cong. Endocrinol. P2-35(Abstr.).

33. Finegood, D.T., L. Scaglia, and S. Bonner-Weir. 1995. Dynamics of $\beta$-cell mass in the growing rat pancreas: estimation with a simple mathematical model. Diabetes. 44:249-256.

34. Habener, J., and D. Stoffers. 1998. A newly discovered role of transcription factors involved in pancreas development and the pathogenesis of diabetes mellitus. Proc. Assoc. Am. Phys. 110:12-21. 